\title{
Accessible authentication: dyslexia and password strategies
}

Karen Renaud

Graham Johnson

Jacques Ophoff

This author accepted manuscript is deposited under a Creative Commons Attribution Non-commercial 4.0 International (CC BY-NC) licence. This means that anyone may distribute, adapt, and build upon the work for noncommercial purposes, subject to full attribution. If you wish to use this manuscript for commercial purposes, please contact permissions@emerald.com'

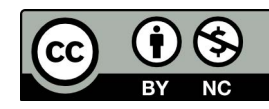

(C) the authors. Published in Information and Computer Security. Published by Emerald Publishing Limited. 


\section{Emerald Information Management}

\section{Accessible Authentication: Dyslexia and Password Strategies}

\begin{tabular}{|r|l|}
\hline Journal: & Information and Computer Security \\
\hline Manuscript ID & ICS-11-2020-0192 \\
\hline Manuscript Type: & Original Article \\
\hline Keywords: & Dyslexia, Authentication, Passwords, Accessibility \\
\hline \multicolumn{2}{|l}{} \\
\end{tabular}

\section{SCHOLARONE \\ Manuscripts}




\title{
Accessible Authentication: Dyslexia and Password Strategies
}

\author{
Karen Renaud, Graham Johnson, Jacques Ophoff \\ School of Design and Informatics, Abertay University \\ cyber4humans@gmail.com,g.johnson@abertay.ac.uk,j.ophoff@abertay.ac.uk
}

\begin{abstract}
A significant proportion of the world's population experiences some degree of dyslexia, which can lead to spelling, processing, sequencing, and retention difficulties. Passwords, being essentially sequences of alphanumeric characters, make it likely that dyslexics will struggle with these, even more so than the rest of the population. In this study, we explore the difficulties people with dyslexia face, their general experiences with passwords, the coping strategies they use, and the advice they can provide to developers and others who struggle with passwords. We collected empirical data through semi-structured interviews with 13 participants. Thematic analysis was used to provide an in-depth view of each participant's experience. Our findings are structured around four research questions, encompassing password issues, authentication systems, and broad experiences of living with dyslexia. We conclude by highlighting the need to enhance the accessibility of authentication to accommodate the needs of people with dyslexia, who are increasingly required to access a variety of personalised and work information and online services. The main contribution of this paper is to provide evidence related to the inaccessibility dimensions of passwords as an authentication mechanism, especially for dyslexics, and to recommend a solution direction. Our aim was to highlight the fact that research into viable, pragmatic alternatives is urgently required if legal accessibility requirements are to be met.
\end{abstract}

Keywords: Dyslexia, Authentication, Qualitative research, Passwords, Accessibility

\section{Introduction}

We increasingly inhabit the online world, now that the 2020 SARS-CoV-2 pandemic has moved education and work online, requiring more authentication and technology use than ever before. The ability to create, retain and enter passwords in the online context requires a range of cognitive skills. These include literacy, the ability to focus, creativity, problem-solving, decision making, attentional abilities, and the ability to keep secrets [33].

One specific disability, dyslexia, is bound to exacerbate the difficulties experienced by the rest of the population when it comes to passwords. Dyslexia is defined by the International Dyslexia Organization [24] as: “...a specific learning disability that is neurobiological in origin. It is characterized by difficulties with accurate and/or fluent word recognition and by poor spelling and decoding abilities... Secondary consequences may include problems in reading comprehension and reduced reading experience that can impede growth of vocabulary and background knowledge."

The first challenge facing any password user is the need to create a password that satisfies all the requirements imposed by the online service being used e.g., minimum length, upper case, lower case, and including a special character. Newly created passwords have to be retained for future use. At some future time, they have to reproduce the password perfectly, mentally tracking the position of the character in the password as they type. It should be borne in mind that memories are not stored in snapshot format in the brain- we reconstruct concepts as and when we need to recall them [10]. If 
dyslexia causes the password to be reconstructed with even the slightest difference from the original, authentication will fail.

We wanted to explore the difficulties those with dyslexia experience with passwords, as well as its close cousins: verification and confirmation codes, one-time passwords and PINs. We needed to carry out an investigation to reveal the coping skills they deployed, and the strategies adopted, given that many dyslexics term themselves "compensated dyslexics" due to their ability to engage in a variety of strategies to mask their dyslexia [11].

The purpose of this research is to reveal the lived experiences of dyslexics in engaging with all kinds of alphanumeric authentication mechanisms. Section 2 reviews the related literature. Section 3 lays out our research methodology and the major research questions we sought to answer via our usercentred approach. In Section 4, we present our findings, and Section 5 reflects on and discusses these. Section 6 concludes the paper with suggestions for further research.

\section{Background Literature}

The research literature into dyslexia, which spans many years, is extensive and occasionally contradictory, with continued debates, especially in the domains of definition and diagnosis. As Kirby [25] points out: "it is useful to think of dyslexia as a both an ongoing psychological diagnosis and a social construct, with all that entails" (p.59). Some researchers question the existence of dyslexia, due to the challenges of diagnosing it with any degree of confidence [18]. The fact that there is no such thing as "typical" dyslexia [6] probably contributes to this difficulty, something that Wagner et al. [44] also emphasizes.

Powell et al. [32] consider dyslexia to be a manifestation of a mismatch between overall cognitive and language ability. They point out that the mismatch and extent of disability varies from person to person. Most experience difficulties in processing sequenced symbolic information [30, 23], manifesting in poor handwriting [39], spelling [20] and reading difficulties. They sometimes have poor short-term memory abilities, and might experience challenges with information organisation. On the other hand, many are particularly strong at visualisation, spatial awareness, creativity, and lateral thinking [45].

The difficulties experienced by dyslexics, both diagnosed and undiagnosed, are evident to anyone working in early education where the issues often first become evident. Such difficulties can have a profound impact on dyslexics' everyday lives, as Phoenix_Rose_13 describes on Reddit [31].

Consider how strings of characters and numbers pervade our lives. Figure 1 shows a gift card, as an example, which requires the recipient to enter a full 18-digit card number when redeeming it online. This is challenging, because the numbers have not been chunked, and many people will make mistakes as they enter this number, with dyslexics likely to find it a particularly error prone process. 


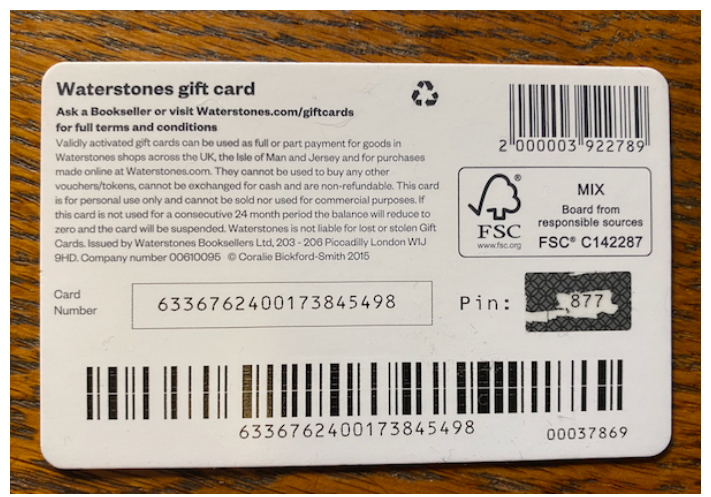

Figure 1: Everyday use of strings of digits (without chunking)

\section{Tools and Guidelines}

There is evidence that some dyslexics make use of spellcheckers to alleviate or correct the errors they might make in other web activities [4]. Morris et al. [29] explored the impact of dyslexia on web searching, given the need to be able to spell and read efficiently, and recommend enhancing readability into search interfaces to help dyslexics.

Powell et al. [30] suggest a number of guidelines for the design of websites to accommodate those with dyslexia. However, these are not necessarily applicable to authentication design where passwords are the dependency. De Santana et al. [17] derived a number of guidelines to inform the design of accessible websites for dyslexics, which include advice about navigation, colours, and layout but do not address authentication. Aziz and Husni [3] publish guidelines for enhancing the readability of text for dyslexics. The guidelines that can apply equally to passwords include: (1) text should be bite-sized and be a list of structured words, and (2) avoid continuous prose. Shih et al. [38] have attempted to address the core elements of the interaction design in terms of fonts, ordering, colours and contrast, and so forth, as do, for instance, UX Movement [41].

\section{Password Usage}

Creating, remembering and entering passwords requires skills that many dyslexics tend to struggle with [35]. Helkala [22] explores the dimensions of these difficulties, which include needing to re-enter passwords due to spelling mistakes during entry, a tendency to choose weaker passwords due to reduced password search space, and increased time used for authentication, as compared to those who do not have dyslexia.

Other opportunities to explore authentication options, for example the recent design of an ATM (Automated Teller Machine) interface to accommodate dyslexics [38], have not focused upon 
PIN authentication accessibility. Alternatives, such as biometrics [12] or alternative visual approaches (e.g. $[14,15])$ have also been proposed but these have not really gained traction in industry thus far.

\section{Related Research}

Renaud et al. [37] carried out a systematic literature review of the literature related to dyslexia and passwords. Within dyslexia research, they identified a number of themes, as shown in Figure 2. There was very little research related to the challenges that passwords pose to dyslexics with some notable exceptions [22]. There were a few studies advancing alternatives, such as musical passwords [21], but these had not been evaluated by dyslexic user groups. They did not find any studies which reported on what dyslexics themselves had to say about their experiences with passwords and a range of other alphanumeric character strings that everyone uses in their day to day lives.

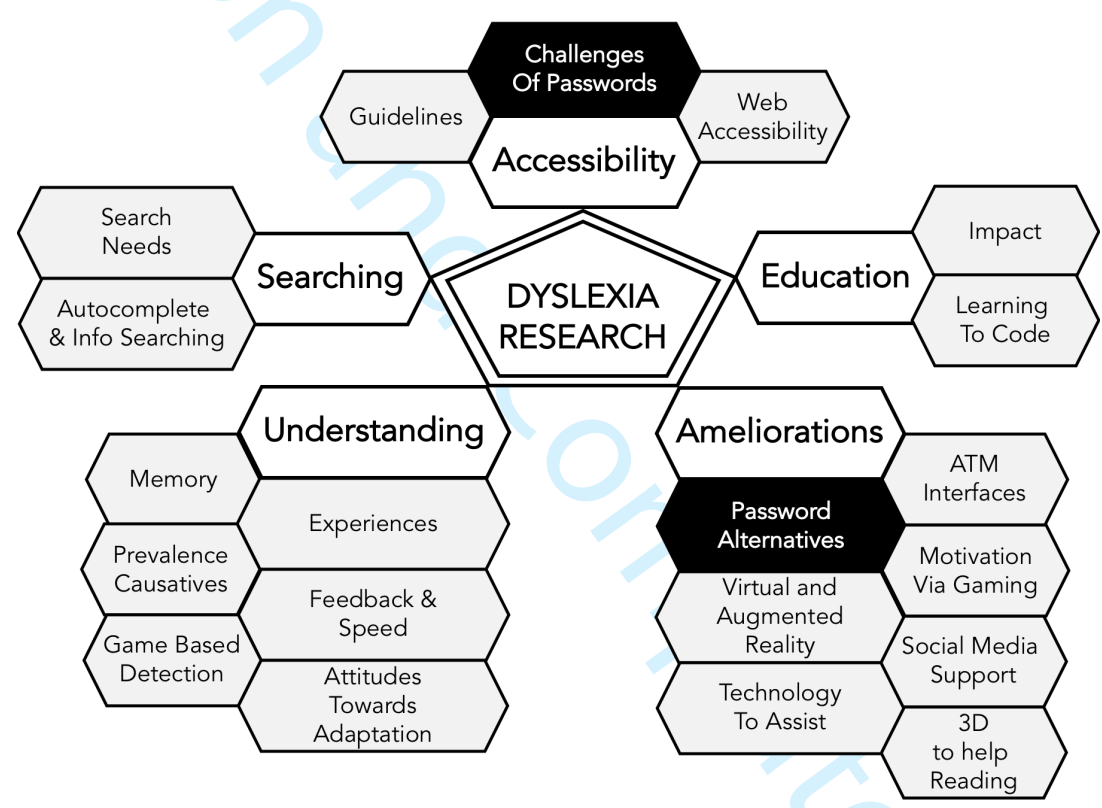

Figure 2: Range of Dyslexia Related Research (Password Topics Highlighted) [37]

\section{Accessibility}

We have had over two decades to resolve the tension between security and usability, initially highlighted by Adams and Sasse [1]. The addition of accessibility, as an extra dimension, undeniably adds complexity to the design process [37]. The tensions between these three dimensions are likely to compete for prominence in the designer's mind. A set of design guidelines would be particularly useful to help them to balance the three.

Guidelines for accessibility, specifically focusing on the web, have been available since the mid1990s. In this context, accessibility means that equal access must be provided to information and 
communication technologies, and opportunity, to people with diverse abilities. This is especially relevant on the web, where barriers to communication and interaction that people face in the physical world can be addressed through good design. At the same time, this also benefits users without disabilities [47].

The de facto standard for web accessibility is the Web Content Accessibility Guidelines (WCAG) published by the Web Accessibility Initiative (WAI) of the $\mathrm{W}_{3} \mathrm{C}$. The WCAG is organised around four broad principles of accessibility [48]:

- users must be able to perceive information and user interface (UI) components using their senses,

- UI components and navigation must be operable using interactions users can perform,

- information and the operation of the UI must be understandable, and

- content must be robust enough to be accessible by a wide variety of (assistive) technologies.

Each of the principles are elaborated through guidelines which provide specific ways to make content more accessible, testable success criteria to evidence conformance, and informative development techniques (e.g., code examples).

The current version is WCAG 2.1, which was published as a W3C Recommendation in June 2018. However, despite its comprehensiveness there isn't any substantive reference to authentication. Only one instance can be found: in reference to providing users enough time to read and use content, the ability to continue an activity after re-authenticating an expired session is specified as a success criterion (2.2.5). This gap may be addressed in the next version of the guidelines (WCAG 2.2) which is currently available as a Working Draft published in August 2020.

WCAG 2.2 introduces a new success criterion called ‘Accessible Authentication' (3.3.7). This specifies that "for each step in an authentication process that relies on a cognitive function test, at least one other method is available that does not rely on a cognitive function test" [49]. In this context, a cognitive function test could refer to remembering a username and password (or any other secret used for authentication). The other authentication method must not be a cognitive function test, for example a password manager automatically filling in credentials, using a device (e.g., with biometrics), or using a third-party login provider. In the case of multi-factor authentication, all steps should comply, i.e., there should be an authentication path which does not rely on cognitive function tests. With this in place "people with cognitive issues relating to memory, reading (e.g., dyslexia), numbers (e.g., dyscalculia), or perception-processing limitations will be able to authenticate irrespective of the level of their cognitive abilities" [49]. A good example is provided by Fuglerud and Dale [19]. 
Regardless of the challenges this will pose to software engineers, accessibility cannot be ignored. Some estimates suggest that up to $20 \%$ of English speakers suffer from a form of dyslexia [28]. Given the fact that, at least in the European Union, websites are required to ensure accessibility, and as the World Wide Web Consortium ( $\mathrm{W}_{3} \mathrm{C}$ ) advises [42,43], we cannot ignore the fact that passwords and dyslexia is likely to be a problematic combination. Spellcheckers [35] and other assistive tools $[30,2]$ are not designed to alleviate password-related issues, nor are electronic readers useful in this respect [34].

The United Nations Convention on the Rights of Persons with Disabilities, adopted in December 2006, is the first international legally binding instrument that sets minimum standards for the rights of people with disabilities. The European Union has an accessibility act which requires those delivering products and services to accommodate the disabled. In addition, accessibility is commonly addressed at national level, such as the UK's Disability Discrimination Act of 1995, which requires websites to ensure equality in access to people with all kinds of disabilities. To meet UK government accessibility requirements a digital service must meet WCAG 2.1 guidelines [50]. Accessibility is clearly a legal mandate though the extent to which websites actually comply is unclear [26].

Numerous studies have highlighted accessibility issues that impact people with different kinds of disabilities. However, only a few of these have focused on dyslexic users specifically [17, 27]. A variety of guidelines and resources that can improve web accessibility for dyslexic users exist, though these are often targeted at other disabilities (e.g., screen readers blind users). For example, the UK Home Office [51] list specific design guidelines for users with dyslexia which include do's and don'ts of text and image use, document layout, contrast settings, and content simplicity. In contrast, Dyslexia Scotland [52] describes how ICT can help address the difficulties associated with dyslexia. They point out text-to-speech and speech-recognition software, spellcheckers, using virtual overlays (to reduce visual stress), learning touch typing, mobile apps, and smart pens. From these examples it is evident that a large variety of coping strategies exist.

An analysis of guidelines relevant to dyslexia suggests that such users could be a prominent indicator group for web accessibility, deserving of more attention [27]. It can also be argued that designing dyslexia-friendly websites will also enhance usability for non-dyslexics. Although neglected, authentication methods also need to improve accessibility, in addition to paying attention to security and usability design considerations, to accommodate the needs of those with disabilities.

\section{$3 \quad$ Research Questions and Methodology}

In this section, we describe our approach to the qualitative field research we conducted with people with dyslexia. Our aim was to ascertain the nature and extent of the influences of dyslexia on password usage in general. We felt it important, having reviewed the extant and varied literature pertaining to 
this domain, to conduct primary research with those whose lived experiences of dyslexia can develop and inform a richer understanding of its impact on a range of online activities.

Adopting an empathic and qualitative approach, as opposed to a formal survey, was a given due to the nature of the participants. People with dyslexia, and similar language difficulties, are typically not at ease with extensive traditional survey approaches. As noted by Zambo [46] and Deacon et al. [16], qualitative approaches can provide a significant and valuable insight into the nature of the lived experience.

Due to the SARS-CoV-2 pandemic, all fieldwork was conducted on-line. After deliberation, we came to the conclusion, given our research questions and the nature of the respondents, that a semistructured interview approach was most appropriate. We envisaged on-line videography or remote interview depending upon participant's preferences, with a requirement of audio recording to support later analysis. Prior to data collection, the research investigation was submitted to, then reviewed and approved by our University's research ethics committee.

Recruitment of participants was via a series of announcements on local university information boards, community newsletters and social media. We had attempted to recruit via national organisations in the dyslexia area, but this did not prove so successful, in comparison with the social media approach. Potential participants were told that we were carrying out an exploratory study of people with dyslexia and their experiences with passwords and similar security and authentication steps. Individuals then contacted the research team and were invited to take part in the interview stage via email. Study information and informed consent forms were provided in written and audio (spoken) format in order to ensure full understanding of the commitment. Interviews lasted up to thirty minutes, and were preceded by confirmation of audio consent of recording.

We interviewed 13 participants, all unpaid volunteers, and chiefly from the United Kingdom. Nearly all interviews were conducted via Zoom ${ }^{\mathrm{TM}}$. All authors were involved in the interviewing stage, following a pre-determined interview schedule. Within the informed consent form, we asked for basic demographic information, namely indications of dyslexia diagnosis, age and gender, occupation and educational level.

The structure and the content of the interview protocol was informed by the four research questions and associated contextual information. We had agreed, in advance, that relevant tangents, and in particular accounts of their broad experiences of living with dyslexia, would also be captured within the interview. The four research questions (RQs) that will allow us to satisfy the aims of this study are:

- RQ1: To what extent, if any, do dyslexics have difficulties accessing IT via common authentication mechanisms such as passwords? This question was designed to gauge the extent of their engagement with technology 
- RQ2: What advice and/or aids, if any, are used in this context, and what strategies are adopted or attempted by those with dyslexia (if having difficulties with passwords and similar)? This homed in on the specific approaches and aids that our participants have used.

- RQ3: In the view of dyslexics, what are the most difficult elements of password processes, and why? This question targeted the ways in which people deal with passwords, and probed tactics for creating and remembering passwords.

- RQ4: What advice or suggestions do those with dyslexia have for the designers of password and authentication systems? This question attempted to gain insights into what people with dyslexia might recommend to those designing and improving authentication systems.

\section{Participants}

The 13 participants $(7 \mathrm{M} / 5 \mathrm{~F} / 1$ preferred not to say) came from a range of age groups (under 30:2, 3140:4, 41-50:3, over 50:4), spanning a variety of different occupations including retail assistant, builder, chef, tutor, dog trainer, student and retiree. Participants exhibited a range of educational achievement from high school to postgraduate, and the majority had been diagnosed or certified as dyslexic, although not always at school. Most were UK residents, with the majority in Scotland, probably due to the scope and range of our recruitment efforts. Table 1 summarises the basic participant profiles.

Table 1: Participant Profiles

\begin{tabular}{|l|l|l|l|l|}
\hline Id & Gender & Age & Educational Level & Diagnosed? \\
\hline $\mathbf{1}$ & Male & $31-40$ & High School & Yes \\
\hline $\mathbf{2}$ & Male & Over 50 & High School & Yes \\
\hline 3 & Male & 30 or under & High School & Yes \\
\hline 4 & Male & Over 50 & High School & Yes \\
\hline 5 & Male & Over 50 & High School & Yes \\
\hline 6 & Male & $41-50$ & Graduate & Yes \\
\hline 7 & Female & $41-50$ & Graduate & No \\
\hline 8 & Female & $31-40$ & Post-Graduate & Yes \\
\hline 9 & Female & $41-50$ & Post-Graduate & Yes \\
\hline
\end{tabular}




\begin{tabular}{|l|l|l|l|l|}
\hline 10 & Female & $31-40$ & Post-Graduate & Yes \\
\hline 11 & Male & Over 50 & Post-Graduate & Yes \\
\hline 12 & Female & $31-40$ & Graduate & No \\
\hline 13 & Prefer not to say & 30 or under & Graduate & Yes \\
\hline
\end{tabular}

\section{Analysis Approach}

The four RQs helped drive the items to be covered and the general structure of the interview protocol. In addition, we sought to ensure we had relevant context, and, importantly, concluded the interview sessions with a request for perspectives on the nature of research approaches with those with dyslexia, where we asked for feedback on the method used and suggestions for improvement.

We adopted a saturation approach to the interviews concluding when there was a substantial degree of commonality and repetition. Across the 13 interviews, we witnessed a set of similar comments and observations, and data patterns, adopting a similar approach to Braun and Clarke [7]. The transcriptions of verbatim interview data were analysed using a commercially available qualitative analysis software tool, NVivo $12^{\mathrm{TM}}$. Themes and codes were contrasted between separate analyses. Those themes and issues raised in our initial review of the literature and area overview [37] helped to inform this emergent qualitative analysis. The following section draws upon the themes and common points of view, from the perspective of the four research questions.

\section{$4 \quad$ Findings}

\section{Education and Employment}

Participants related significant experiences from their school years. For many, especially the younger age groups, the impact of dyslexia on their lives has been significant, with their educational experience often overshadowed by their difficulties. Several commented, with a level of gratitude, on the measures and support in place during primary, secondary, and higher education. Participant 8 noted:

"I had a member of the support department sit next to me ... to help me with my work ... everyone could make sure that these tools were in place."

"And lots of strategies in high school. I had scribe, use of a computer, Belkin spell checker, use of a laptop in college ... a Dexter Dictaphone, Dragon and read and write gold, things like that yeah." 
It is important to note that our participants had a range of educational and work accomplishments, with one clearly viewing their publication of a book and doctorate as challenges he had overcome, despite his difficulties. Within the work context, some had experienced difficulties attributed to a lack of understanding, whilst others had declared their difficulties and received support from employers. For example, Participant 6 was pleased to note that:

"...my organisation has been really good about disabilities - the HR department has engaged with dyslexia societies to support employees"

All acknowledged that their dyslexia had had a significant and constant impact on their lives, not just in educational and work settings, but also in communication with family and friends.

\section{Life Impact of Dyslexia \& Coping Strategies}

It became clear that the types and experiences of dyslexia, or at least its impact on tasks involving reading and writing, were varied. Some said that the effects usually manifest in reading, where words or letters appear to move, or reading under pressure (and mixing up words) and writing (in particular with spelling and order). However, some participants also mentioned an emotional impact of feeling 'stupid', and being 'taught to hide' from an early age. Some felt strongly that this 'label' in itself was a differentiator at school and beyond, whilst others were keen to point out how they had embraced it as a strength and focussed on how a different way of viewing things provided an advantage.

The conversations about strategies, skills, and aids ranged from those who had simply got on with it', to those with many strategies and tactics depending upon context. Many made reference to technical aids as a way of coping. Such support mechanisms included spelling aids, spellcheckers, autocorrect and predictive text. For instance, Participant 5 recalled that:

“...one of the things I got in 1997 was a Franklin Bookman word speller and for 20 years it was superb. Spell checkers have got better on computers. But with this checker it would come up with the word I was looking for. And, if I wasn't sure, you could go to the thesaurus and you could get the word. Now a couple of buttons stopped working. That's when I found computer software wasn't brilliant, but it's getting better. That was a brilliant piece of kit."

Others spoke about using speech software or domestic technology such as digital voice assistants to check and search for things. However, the majority called out spelling and text-based tools. Participant 10, who employed this approach regularly at work, mentioned text expander software: 
"I usually use text expanders to make sure that I'm so that I've checked it before.” ... “ Text expanders... if I were to type that out it would take a long time for me to check myself, so if I if I've already written it, and checked through it, then it just sort of expands itself. So it works it works in pretty much any application, it works online, and that kind of stuff, so it just sort of populates things."

Some, as an aid to organising information, also saw having two screens as an advantage. For example, Participant 7 said:

“But I have two screens and I can't do without them. I can't remember information so I have to have it displayed on the one screen to copy it across from one screen to another"

A few were well-versed in the use of filters, and different fonts, and spoke about customisation across a number of different sites, platforms and devices, noting their occasional frustrations with some browser and system incompatibilities.

"Yes, so basically I, well, for my, it's a Kindle I've got, with adaptations for it - where it uses to open dyslexia font and I can put a coloured tint to it increases the contrast. And, for me the colour purple works really well too kind of fix things in place so I can read it without jumping around inadvertently, or things flickering. Yeah. I also use plastic tinted overlays for written work, and again it kinda fixes things in place for me." (Participant 6)

The use of filters, and of printing information, and of taking more time to go through things, cropped up many times. Filter colours and preference differed, and, for some, these approaches had had no meaningful impact. Participant 12 spoke about their work situation, working within a team, and the variety of systems used.

"Unfortunately, we're hot-desking all the time, so I can't really tape anything to monitors or anything like that. What I sometimes do is print some stuff off, and ... or sometimes even highlighting the text so it shows up blue - that sometimes helps me a wee bit."

On screen overlays, several noted their own, individual approaches: 
"I think Dyslexia Scotland.... I also got diagnosed with scotopic sensitivity. I get very tired reading - an orange overlay helps - definitely less straining on the eyes it improves with age anyway. The words move less on the page" (Participant 6)

“... amber kind of coloured plastic sheet if they're reading. I found that I didn't get on with that. Um, can I just tell you something - it was that ambassador or something that's recommended to them yeah, it was at their school - amber plastic see-through sheets." (Participant 11)

\begin{abstract}
Doing Research with Dyslexics
We also asked about our research approach in order to ascertain the participants' views of the most appropriate way of attempting to understand the challenges faced by those with dyslexia in the security domain. They were able to confirm that a qualitative approach, one that relies upon conversation as opposed to surveys, was most suitable. Many were complimentary about the way we had structured the research, and considered this research an important and meaningful topic. Some wanted to make sure that any future research allows enough time, more time than would perhaps normally be set aside to carry out tasks.
\end{abstract}

\title{
RQ1: To what extent, if any, do dyslexics have difficulty accessing IT via common authentication mechanisms such as password usage?
}

Most participants were active users of technology, including smartphones, computers, and tablets. Some reported computing equipment and devices used both at work and at home. They used these daily, exhibiting a measure of comfort and proficiency with these specific technologies. One participant commented on their digital literacy, stating that:

"I’m fairly tech literate. I am usually the person that people go to for their tech problems and things like that." (Participant 12)

However, most participants faced problems on a daily basis. Participant 7 stated "... so many times a day I couldn't count it."

While it is often difficult to estimate the scope of the difficulties experienced by others, participants speculated that most dyslexics would have difficulties with technology, especially with passwords. Participants thought that at least 80 percent of dyslexics would experience issues. Some suggested that every dyslexic would struggle. However, experience with technology, and the severity of an individual's condition would influence this. 


\section{RQ2: What advice and/or aids, if any, are used in this context, and what strategies are adopted or attempted by those with dyslexia (if having difficulties with passwords and similar)?}

Participant 8, as did several others, used a system of (safely written down) passwords and then rotated and reused whenever possible. On the nature of dyslexia influencing password difficulties, feeling a little 'stupid' and the like, Participants 12 and 10 and 9, respectively, commented:

"I think it affects it quite a bit because I don't have that kind of memory for, you know, where somebody else might be able to go, you know, right, it was, cat spelt with an 'a' or it was alpha, spelled with an 'at' sign. My brain doesn't seem to remember that. And then occasionally, I'll reverse things, you know, reverse things, or reverse letters and things like that I won't notice it, and then I put the same thing again and again, and then suddenly it works, I can't remember, and I probably reversed something haven't I? So, I think ... probably I have a shorter processing memory, apparently, so I think that sometimes can make things quite difficult.”

“... I used to live in China, so I set up, well, I was asked, in the bank, to set up a password. So, I set up a password but then like a few minutes later I couldn't remember it. Because I hadn't written anything down... But yeah it was a very noticeable and it was really embarrassing because, you know, you're, you're, in a bank and you've just been asked to do something and everything's happening, in another language, and it was all quite stressful anyway and then so you know it seems like a really stupid mistake. But, yeah I just couldn't do what I normally do to avoid it."

"But it's the level of frustration and upset that it causes me. My natural default was 'how stupid can I be' that I cannot remember a set of passwords in you know a list of ten!”

On the question of password creation strategies, we came across several and a reasonable amount of consistency. It was interesting to hear about password creation strategies, especially where we were clear that we did not want to hear any password, but rather to gain an understanding of their approach. Given the apparent difficulty of recalling passwords, it seemed as if a great deal of effort had been made in this area, with many being quite pleased with their strategies. There was a variation of folk using a 'long stem plus required characters', others who favoured a phrase or saying and used this (or these) as the core, and others who made use of other languages, one even having used Klingon and Elvish elements within passwords. On strategies, and some termed them a 'formula' or a known base. Recycle or reuse appeared common, for instance: 
"I use something familiar like a name or a number I already know" (Participant 4)

"I'll be really honest with you - I rotate them, but you'd have to work quite hard to figure out how I rotate them, if that makes sense.” (Participant 9)

We heard about avoiding certain situations, as a strategy for coping, and this covered a number of instances. A couple mentioned the common situation in a car park and the registration step. This everyday scenario was seen as a conclusion to illustrate difficulties.

“... In the car park, you have to put your registration in. Used to be, you could put in the first two letters and a picture of your car would come up. Now you have to enter your full registration, and I transpose the numbers, and get it wrong. Now, I just don't go back to that supermarket. It's my way of coping. I would worry if I got it wrong, but now if they fined me, I would simply say I have a disability and refuse to pay." (Participant 5).

We included PIN authentication in our discussions. Several mentioned that they had relatively elaborate ways of safely storing their codes, PINs, and passwords, often constructing a system for recording or writing down these valuable 'keys'. The following illustrate such approaches:

"I have an old business card and on the back are both real and fake phone numbers and within those I have the passwords" (Participant 6)

"When we had numerical passwords I would write them in my address book, so that they looked like a real phone number. Always put 01382 in front. I camouflaged it." (Participant 5)

"Anyway, so there's no way on there if I'm remembering that, um so uh, I put these numbers in my phone. So, I pretend it's a person, and I write, I make up a phone number, and the last four digits of that phone number are the pin number." (Participant 10)

In summary, we learned a great deal from our research participants on the question of strategies. Many were keen to point out that they believed that because of the difficulties they had experienced, having a habit or system was especially important.

In terms of the advice provided by others, whether family or friends, or dedicated organisations, we were rather surprised to find that almost no one had received or followed advice concerning dyslexia in relation to password security. However, some had seen advice or had come across general guidance, for example: 
"I saw YouTube videos about Last Pass, so I decided to use it" (Participant 1)

"Nobody ever has given me tips, other than that they [passwords] all have to be different" (Participant 7)

\section{RQ3 - In the view of dyslexics, what are the most difficult elements of password pro- cesses, and why?}

When we asked about the difficulties encountered with passwords, there was an overwhelming consistency in responses, with many of these issues being familiar. Participants commented upon the complexity, the special requirements, such as upper and lower case and special characters, limited length of passwords, expiry policies enforced by some systems, (mis)spelling issues, their storage approach, and their reuse strategies. We queried not only passwords but also authentication (as in two factor) codes, and everyday 'passwords' such as banking PINs (personal identification numbers), and access and entry codes. On the nature of spelling and its repetition, some felt challenged, such as Participant 5:

"I spell them incorrectly. Especially if you have to type the password and confirm it, I can't do it the same twice."

Others felt strongly about mandated complexity:

"It's a struggle to meet the requirements and also remember the resulting password. But if then I'm asked to add exclamation marks, figure, shapes, or stars, that's a troublesome one. Especially once you've done it and they say this is not secure enough!” (Participant 11)

The majority spoke about password length, with two perspectives. Some were firmly of the view that passwords ought to be allowed to be longer - in this case, to enable people to have known, familiar phrases as password stems. In contrast, others stated that shorter passwords were easier to recall. Participant 9 made the point that:

“... over a certain length, and under a set, or you know a maximum number of characters, they are usually the ones that floor me the most."

On the question of complexity and associated requirements, Participant 2 and 13 respectively commented that: 
"You write a password and it doesn't accept it. So, you change it and it still doesn't accept it. Bigger, longer. Okay, still doesn't accept your password, you don't see why, and you're wondering what it's on about."

"Yes, it [password complexity requirements] does make things slightly more difficult. Although I do understand the need for Internet security and I do appreciate it. I find that as passwords have got more complex, especially in the last five years, that my ability to manage my passwords has went down. As different variations of the same password, as I talked about, have emerged and I have struggled to keep track of that."

Several mentioned the spelling challenge of passwords, and on being prevented from using the same password again, for instance:

“... I would have the password spelt differently to what people would have that word spelt correctly." (Participant 1)

“...there's a system that I can use when my password expires - it often takes me a while to find one that's not been used already. But I get there eventually.” (Participant 8)

It was noted by several participants that other factors and conditions were playing a role in combination with their dyslexia:

"I have dyscalculia which is about numbers ... a massive, massive issue. I struggle every day to get numbers in the right order." (Participant 7)

"I mostly have visual disturbances, so things flicker and if I haven't memorised how to spell a word I can't sound it out or anything like that...” (Participant 12)

Feelings about account lockout, password resetting and fall-back steps, such as security questions and the like, came to the fore when participants spoke about the degree of frustration they experienced, and sometimes embarrassment, contrasting this, in their view, with those who do not have dyslexia. The interpretation of such events varied between participants where some felt that that there is a notion of proving identity, others felt that their general competence had been challenged. 
"This morning I locked myself out of the online register for the school. Then, I managed to lock myself out of the office portal. There's this like tech portal - there's no technician in school anymore because of COVID, so we have to log the call with an online ticket... and then the technician supports you, but over the phone. They said to me, you know, we'll ask you security questions. That's fine, except when they say can you give me the first digit and the fourth digit of your password!” (Participant 8)

"Um, I think the funniest one was our office, when it's back open, has a keypad entry for the staff area. Yeah, one day with my manager and went 'what are you doing at the door'? I'm like, 'I can't remember what the number eight looks like'. And she was like 'what?' Because she doesn't get it." (Participant 8)

Some had common strategies for coping with the difficulties in remembering and managing a number of passwords:

"It's remembering the password. We don't have good memories. You have to write it down" (Participant 4)

"I have a little book - and I write all my passwords in it” (Participant 1)

"For several years I kept a written document of all of my passwords and information like it. So I would write down the site name, let's say Facebook. I'd write down the email that's connected to that. I'd write down the password that I was using. I would write down the security question. It did help me manage it for a good few years. However, I stopped using that and I essentially feel like I'm in the deep end now. I have already committed to not using it anymore and it has caused issues." (Participant 13)

As expected, many stated that, even though they knew it was advised against, they would use the same password wherever possible. On using a password manager, and storing passwords, research participants commented:

"LastPass helps me. But I also try to remember it, in case LastPass goes wrong, then I can still remember my passwords to the apps. LastPass is a fail-safe. If I regularly log in, I don't struggle. I usually conquer it by typing it really slow, to make sure it is right” (Participant 3) 
"Most of the time I'll ask my computer to remember my password because otherwise I'm going to just spend reams of time going through probably the sets of passwords that I have." (Participant 9)

It appears that regular usage, where patterns almost become semi-automatic, are less problematic, and of course, those used less frequently prove to be difficult, as noted by a couple of participants:

“...it'll be the same with you know door pass codes etc. If I'm going through that door regularly, I will remember that number. That's um I've got a couple of passcodes that are up to six digits long, and because I'm using them you know most days not a problem at all. If it's gonna be rarely, you know once a month, it'll take me a good few months to remember that pattern, and I have to make a point of wanting to, but I think that's probably the same for every human being. I have to say 'right now I'm going to learn my pattern okay'. I know it's done and it'll be there." (Participant 9)

"Um no, yes, um and my example would be the long key code secure door locks... to get through to somewhere else, um if they were all numbers - easy, I'd remember them - just boom boom! Easy. If they were numbers and letters, I would remember the shape, the yeah, the space shape.” (Participant 11)

\section{RQ4, was - What advice or suggestions do those with dyslexia have for the designers of authentication systems?}

Within this area of the investigation, we asked about their experience of and opinion about some alternatives such as biometric verification technologies. The majority of participants used or had used biometrics before and, for the most part, had had relatively positive experiences. The increased deployment of finger- and thumb-print, as well as facial recognition on contemporary smartphones, has had a positive effect. However, some mentioned the frustration of a fall-back PIN or password, and others were concerned about privacy. In general, biometrics were seen as a reasonable alternative to passwords.

We also probed as to awareness of alternatives such as graphical and musical passwords. Nearly all had little knowledge of these. Some made mention, equating pictorial verification approaches, with the likes of the 'I am not a robot' type CAPTCHAs, and picking out pictorial elements within a picture array, when registering for a service, site or subscription. On musical and graphical approaches: 
"That's quite ingenuous. I usually remember small details and then I can remember things" (Participant 3)

"For low risk systems that sounds great. Interestingly enough, music is one of those things that sticks quite easily. Talked to a friend who is more dyslexic. He can pick up a song and retain it easier than time tables." (Participant 6)

"No, I haven't. But that sounds interesting. That would be really interesting. I do tend to remember a tune probably a lot easier than, you know, a random string of letters. Yeah, to be honest giving people as much options as possible is the best thing, because everybody's, everybody's experience is going to be slightly different, and that's whether or not you have a diagnosis or not." (Participant 12)

With regard to system design, we explored the universal and on-size-fits-all approaches, where many felt strongly that there was no feasible 'one-size' approach. Our dyslexic participants, understandably, made the point that systems should cater for all, and some questioned the experience of the designers themselves.

"I genuinely believe that people are beautiful unique beings, and if anybody manages to find a genuine honest one-size-fits-all that covers the whole of mankind I want to shake that person by the hand. Because they are incredible. And we're all forced, encouraged, to do things that maybe we would choose not to, I'd rather not have a password, but I do understand, that it's for my own protection, that other people are looking to get my data, and that I don't want them to have my data. So yeah, I think humans are far too complex for us to ever find a one-sizefits-all.” (Participant 9)

“... a lot of the strategies I use are really helpful to somebody with dementia. Because it's that sort of processing and working memory. So, if you're making your software more accessible to one group of people, you inadvertently make it accessible to more groups of people." (Participant 12)

Comments were made about not just about the design of systems per se, but also about the processes surrounding them, for instance those of procurement and selection, and on policies determining types of use or implementation. One spoke about their work experience and the way in which systems had been chosen, and the impact that had had upon everyday work practices: 
"So, for example, the NHS mental health services all switched over to specific application for note taking. Our old one was web browser-based, so even though was inadvertently accessible, and it was actually really easy to go and tint things, and change the font - that kind of thing, even though it wasn't built into the actual application. Then we switched over to a new system called EMIS, which is an actual application and it's completely locked down. Because we bought a specific version of it, we can't make any changes to it at all. So the people who, well, I was on one of the secondary em committees for it, for putting it out amongst the wards, and I immediately brought up that I can't customise it, I can't change it you know, I can't make it accessible for me.” (Participant 12)

Interestingly, some also commented extensively on the general usability and interface elements of systems, and not just those aspects concerned with passwords. Some focused upon the need to have a broader understanding of usage, others pointed out the range of people expected to use systems in a variety of settings. We also had comments on the knowledge designers appear to use:

"because I think a lot of people don't have any understanding - and then designers of applications and things like that will make something to somebody's standard which is, you know, if they haven't had people with additional needs in mind, then they are often quite unusable." (Participant 12)

Others pointed out some interface attributes and general design points:

"Yeah, stop trying to be so clever and complicated - show the mistakes you make." (Participant 2)

“...we need a tool that types in dyslexia font, but when you print it uses the usual font" (Participant 6)

With respect to ideas on password 'design' approaches, several participants related this to their own use, and previous and current problems, needs and preferences.

"I don't mind having a password, that's fine. I should be allowed to pick a password that is easy to remember. I'm not talking about 123456 , or a really common word, but something I can put in myself, something I can see, that helps. The fact I can't see it, that's the problem - you have to type it in three times, that helps, repetition helps if I could see it, that helps - just a reveal 
thing.... I am sure there's lots of things but for me it's about laying things out carefully the smaller the writing the more difficulty I have in making out the letters" (Participant 7)

"Can we stop putting a maximum number on it - that would be really helpful. So, I get a minimum number and it's got to have you know capitals, lowercase, some kind of mark in them, and some kind of number, that they can do. Can we stop rigidly saying that they have to maybe be between 8 and 14 characters long and open that up?” (Participant 9)

"And for them not have daft rules. Like, you know, by putting a limit on the amount of letters that you allowed or things like that. That's one of the things I come across very frequently it'll be 'your password has to be you know between 6 and 15 characters." (Participant 12)

"And I think my online banking was possibly one of the worst things for me. Again the examples were strings of zeros, so I couldn't count that, yeah, so, no not friendly but unfriendly, that has caused unnecessary angst, and that's why I thought I'd get involved with this." (Participant 9)

"It would be very customisable. So, I would want to be able to change font, the font size, and I'd want to be able to change whether or not things showed up bold. I'd want to be able to go and add a tint to it, if I needed to, for it to be accessible with online readers, you know, text to speech.” (Participant 12)

General password advice to other dyslexics was also prompted within the interviews, with participants drawing on what works for them, and keen to help others with similar challenges with passwords. For example, in the use of core passphrases and memory strategies:

“...ask what their hobbies are. Look for simply spelt words. Something they have a passion about. Use those words. Sometimes they are not long enough. Then use some words, two or three, to make the pass phrase. I normally put a number at the end. If I put it in the middle but I might forget where it should be.” (Participant 5)

"Try and find a memory strategy that works for you. So, whether or not it's coming up with a visual password that you associate with words. So, I've got a couple of like memory prompts, of drawings, and they're associated with words in a password. So, I'm going to make one up here but you know... er, 'taco cat' for example - it will be a picture of a taco and a cat. Something that means something to me, so somebody else won't have a blooming clue! Cos' it will just 
look like a doodle. And, again thinking of kind of an odd sentence, or something like that, but something you would remember something that you just you know. Stuff like that helps me, rather than like picking a random string of words, and things like that. Where it falls down it's where you get to passwords where they require like a specific amount of words or they put a limit on the amount of letters you're putting in. That's where it tends to get a wee bit more difficult and it falls down.” (Participant 12)

\section{$5 \quad$ Reflection \& Discussion}

Our literature review suggested that dyslexics might experience difficulties with passwords, and our interviews confirmed it. Dyslexics told us about a range of strategies, experiences, and skills they use to navigate a world populated with alphanumeric strings that challenge them and can interfere with the completion of their goals: PINs, parking areas, travel ticket collection, two factor authentication codes and passwords. This is a serious lack of accessibility, experienced by citizens in a country where accessibility is a legal requirement. Along these lines all the interviewees specifically mentioned that the audible version of the consent form as very helpful.

Our participants mentioned being labelled as 'stupid', echoing Berget and Fagernes [5] and talked about negative experiences in school and in their personal lives due to their dyslexia. It was also clear that the global move online in 2020 had exacerbated the challenges they faced because they had even more passwords to deal with than usual.

We now return to each of the research questions:

- RQ1: To what extent, if any, do dyslexics have difficulties accessing IT via common authentication mechanisms such as passwords? We revealed a range of difficulties during password creation, retention (memorizing) and entering when they returned to websites. There was mention of the extra time needed to create passwords, and then to memorise them and to authenticate. If one multiplies this extra time by the number of times the average user authenticates every day, it becomes a significant time sink.

- RQ2: What advice and/or aids, if any, are used in this context, and what strategies are adopted or attempted by those with dyslexia (if having difficulties with passwords and similar)? Some of them resorted to practices such as writing down passwords, or spent far too much time using the password reset functionality whenever they need to provide a password. Others allowed the browser to remember their passwords and many had formulae that they used to come up with passwords, which alos helped them to remember the passwords later. 
- RQ3: In the view of dyslexics, what are the most difficult elements of password processes, and why? They struggled to create passwords because of the complexity requirements imposed by many online services. They also struggled to rememeber and replicate the passwords precisely later. This led to them getting locked out of accounts, and wasting time trying to get password resets. This required them to create a new password, starting off the entire process from scratch.

- RQ4: What advice or suggestions do those with dyslexia have for the designers of password and authentication systems? Our interviewees wanted password alternatives, with biometrics being a particularly acceptable option. They also mentioned password policies imposed by their employers sometimes being overly stringent, and imposing requirements that they found it difficult to meet. All wanted system designers and developers to be aware of the needs of those with disabilities and to design their systems accordingly.

\section{Summary}

Our findings confirm the need for accessible authentication [49]. Our participants all perceived difficulties with cognitive function tests, i.e., authentication with a username and password, or PIN. Our analysis illustrates the range of problems experienced, but also the coping strategies people use. Of concern is that some of these strategies, such as writing down or reusing passwords, will compromise security. However, it is hard to blame users or systems designers when the alternatives are not clear.

Alternative authentication methods, and robust support for them, will greatly improve accessibility for users with dyslexia. Our participants' experiences indicate that biometric verification technologies present a secure, usable and accessible alternative. However, in cases where this is infeasible, an alternative fall-back mechanism must be provided. A possible technique recommended by the $\mathrm{W}_{3} \mathrm{C}$ is to email link authentication [53]: "This technique involves providing an authentication mechanism where the user can enter their email address, and they are sent an email with a link to click. When the user clicks the link in the email, they are directed back to the website and automatically logged in." Indeed, this method of authentication is already supported by current web applications.

\section{Limitations}

There is a possible volunteer bias, as we are dealing with self-reported data including historical and reflective elements, and we are seeking information only from those with self-declared or diagnosed dyslexia. Furthermore, many expressed an interest or curiosity in the relationship between dyslexia and password difficulties, for some a motivation for their participation. Finally, given that our participants told us that dyslexics might hide, it is possible that the experiences of those who do hide are different from those who chose to speak to us and thus were not hiding. 


\section{Conclusion \& Future Work}

These interviews gathered self-reported challenges and successes experienced by dyslexics dealing with passwords, authentication, and related approaches. Overall, we confirmed that passwords have severe accessibility flaws for dyslexics. We were able to provide some key insights which should inform developers who have to authenticate their users.

There is much people-centred work still to be done in this space, in particular to raise awareness of the issues a large percentage of the population face daily, in interacting with the world's most ubiquitous authentication mechanism: the password.

\section{References}

1. Adams, A. and Sasse, M.A., Users are not the enemy. Communications of the ACM, 42(12), $40-46$ (1999).

2. Athènes, S., Raynal, M., Truillet, P. and Vinot, J-L. Ysilex: a friendly reading interface for dyslexics. (2009).

3. Aziz, F. A., and Husni, H. Interaction design for dyslexic children reading application: A Guideline. Pp. 682-686 (2012).

4. Baeza-Yates, R. and Rello, L., Estimating dyslexia in the Web. In: Proceedings of the International Cross-Disciplinary Conference on Web Accessibility, pp. 1-4. (2011).

5. Berget, G., and Fagernes, S., "I'm not stupid”-Attitudes Towards Adaptation Among People with Dyslexia. In: International Conference on Human-Computer Interaction (pp. 237-247). Springer, Cham. (2018).

6. Brachacki, G. W. Z. Towards a multimedia computer assisted careers guidance system for adults with dyslexia. PhD diss., University of Sheffield, 1999.

7. Braun V., and Clarke V. 'Using Thematic Analysis in Psychology.' Qualitative Research in Psychology 3(2): 77-101. (2006).

8. Broadhead, M., Daylamani-Zad, D., Mackinnon, L. and Bacon, L. A Multisensory 3D Environment as Intervention to Aid Reading in Dyslexia: A Proposed Framework. In: Proceedings of the 1oth International Conference on Virtual Worlds and Games for Serious Applications. IEEE, 4 pages. (2018).

9. Chai, J.Y. and Chen, C.J., A Research Review: How Technology Helps to Improve the Learning Process of Learners with Dyslexia. Journal of Cognitive Sciences and Human Development. March, 2(2), 26-43. (2017).

10. Clewett, D., DuBrow, S., \& Davachi, L. (2019). Transcending time in the brain: How event memories are constructed from experience. Hippocampus, 29(3), 162-183.

11. Cole, L., MacFarlane, A., and Makri, S. More than Words: The Impact of Memory on how Undergraduates with Dyslexia Interact with Information. In: Proceedings of CHIIR'20, 14 March, 2020, Vancouver, Canada. 5 pages. (2020).

12. Coventry, L., De Angeli, A. and Johnson, G.I., Usability and biometric verification at the ATM interface. In: Proceedings of the SIGCHI Conference on Human Factors in Computing Systems. Association for Computing Machinery (ACM), pp. 153-16o. (2003).

13. Cullen, E. A Study into Dyslexia and the level of awareness amongst FAS staff at the FAS Training Centre Finglas. PhD diss., National College of Ireland, 2005.

14. De Angeli, A., Coutts, M., Coventry, L., Johnson, G. I., Cameron, D. and Fischer, M. H., VIP: a visual approach to user authentication. In: Proceedings of the Working Conference on Advanced Visual Interfaces, AVI 2002. De Marsico, M., Levialdi, S. and Panizzi, E. (eds.). New York: Association for Computing Machinery (ACM), pp. 316-323. (2002).

15. De Angeli, A., Coventry, L., Johnson, G.I. and Renaud, K., Is a picture really worth a thousand words? Exploring the feasibility of graphical authentication systems. International Journal of Human Computer Studies. 63(1-2), 128-152. (2005).

16. Deacon, L., Macdonald, S.L. and J. Donaghue. "What's wrong with you, are you stupid?" Listening to the biographical narratives of adults with dyslexia in an age of 'inclusive' and 'anti-discriminatory' practice, Disability \& Society, (DOI: 10.1080/o9687599.2020.1815522) (2020).

17. De Santana, V.F., de Oliveira, R., Almeida, L.D.A. and Baranauskas, M.C.C., April. Web accessibility and people with dyslexia: a survey on techniques and guidelines. In: Proceedings of the international cross-disciplinary conference on web accessibility, pp. 1-9. (2012). 
18. Elliot, J. G. It’s Time to Be Scientific About Dyslexia. Reading Research Quarterly, 55(S1), S61-S75, doi:10.1002/rrq.333 (2020).

19. Fuglerud, K. and Dale, O., Secure and inclusive authentication with a talking mobile one-time-password client. IEEE Security \& Privacy, 9(2), 27-34. (2011).

20. Garcia, J.M., The lived experience of adolescents with dyslexia. Master of Science in Communication Sciences and Disorders. University of New Hampshire. (2007).

21. Gibson, M., Renaud, K., Conrad, M. and Maple, C., Play That Funky Password!: Recent Advances in Authentication with Music. In Handbook of Research on Emerging Developments in Data Privacy, pp. 101-132. IGI Global. (2015).

22. Helkala, K., August. Disabilities and authentication methods: Usability and security. In 2012 Seventh International Conference on Availability, Reliability and Security, pp. 327-334. IEEE. (2012)

23. Howard Jr, J. H., Howard, D. V., Japikse, K. C., and Eden, G. F. Dyslexics are impaired on implicit higher-order sequence learning, but not on implicit spatial context learning. Neuropsychologia, 44(7), pp. 1131-1144. (2006).

24. International Dyslexia Organization, Available at: https://dyslexiaida.org/definition-of-dyslexia/, [Accessed on 8 March 2019]. (2019).

25. Kirby, P., A brief history of dyslexia. The Psychologist. BPS. March 2018, 31, 56-59. (2018)

26. Kuzma, J.M., Accessibility design issues with UK e-government sites. Government Information Quarterly, 27(2), 141146. (2010).

27. McCarthy, J.E., and Swierenga, S.J. What we know about dyslexia and Web accessibility: a research review. Universal Access Inf. Soc. 9, 47-152. (2010).

28. Michail, K. Dyslexia: The experiences of university students with dyslexia. Doctoral dissertation, University of Birmingham. (2010).

29. Morris, M.R., Fourney, A., Ali, A. and Vonessen, L., Understanding the needs of searchers with dyslexia. In Proceedings of the CHI Conference on Human Factors in Computing Systems, pp. 1-12. (2018).

30. Pařilová, T. DysTexia: An Assistive System for People with Dyslexia. PhD diss., Masarykova univerzita, Fakulta informatiky. (2019).

31. Phoenix_Rose_13. My life with dyslexia (sorry it's a bit long.) https://www.reddit.com/r/Dyslexia/comments/jcw1rs/my_life_with_dyslexia_sorry_its_a_bit_long/ 17 October (2020).

32. Powell, Norman, David Moore, John Gray, Janet Finlay, and John Reaney. Dyslexia and learning computer programming. Innovation in Teaching and Learning in Information and Computer Sciences, 3(2), 1-12. (2004)

33. Prior, S., Renaud, K., Age-Appropriate Password "Best Practice” Ontologies for Early Educators and Parents. Early Childhood Education Journal. Volumes 23-24. (2020).

34. Rello, L., Baeza-Yates, R., Saggion, H., Bayarri, C. and Barbosa, S.D., October. An iOS reader for people with dyslexia. In Proceedings of the 15th International ACM SIGACCESS Conference on Computers and Accessibility, pp. 1-2. (2013).

35. Rello, L., Ballesteros, M. and Bigham, J.P., October. A spellchecker for dyslexia. In: Proceedings of the 17th International ACM SIGACCESS Conference on Computers \& Accessibility, pp. 39-47. (2015).

36. Riley, C., Buckner, K., Johnson, G., and Benyon, D. Culture \& biometrics: regional differences in the perception of biometric authentication technologies. AI and Society, 24(3), 295-306. (2009).

37. Renaud, K., Johnson, G.I. and Ophoff, J. Dyslexia and password usage: accessibility in authentication design. In: Human Aspects of Information Security and Assurance: 14th IFIP WG 11.12 International Symposium, HAISA 2020, Mytilene, Lesbos, Greece, July 8-10, 2020, Proceedings. Clarke, N. and Furnell, S. (eds.). Cham: Springer, p. 259-268 10 p. (IFIP Advances in Information and Communication Technology; vol. 593). (2020).

38. Shih, M-S., Chang, J-C., and Cheng, T-Y. The design guideline for dyslexics-friendly Chinese ATM interface. In: IC4E '19: Proceedings of the 10th International Conference on E-Education, E-Business, E-Management and E-Learning, pp. 416-420. (2019).

39. Sumner, E., Connelly, V., \& Barnett, A. L. The influence of spelling ability on handwriting production: Children with and without dyslexia. Journal of Experimental Psychology: Learning, Memory, and Cognition, 40(5), 1441. (2014).

40. Wilson, Patrick. Dyslexics, know your brain. https://senmagazine.co.uk/articles/1096-how-your-brain-works-differently-as-a-dyslexic.html (Accessed January 2020). (2020).

41. UX Movement., 6 Surprising Bad Practices That Hurt Dyslexic Users. https://uxmovement.com/content/6-surprising-bad-practices-that-hurt-dyslexic-users/ (Accessed 10 March 2020). (2011).

42. W3 $\mathrm{C}$ - Web Accessibility Initiative. Web Content Accessibility Guidelines (WCAG) 2 requirements and techniques. https://www.w3.org/WAI/WCAG21/quickref/?versions=2.0 [accessed March 2020]. (2019).

43. $\mathrm{W}_{3} \mathrm{C}-\mathrm{Web}$ Accessibility Initiative. Accessibility, Usability, and Inclusion. https://www.w3.org/WAI/fundamentals/accessibility-usability-inclusion/ [accessed March 2020]. (2020). 
44. Wagner, Richard K., Fotena A. Zirps, Ashley A. Edwards, Sarah G. Wood, Rachel E. Joyner, Betsy J. Becker, Guangyun Liu, and Bethany Beal. The Prevalence of Dyslexia: A New Approach to Its Estimation. Journal of Learning Disabilities (2020).

45. Wilson, Patrick. Dyslexics, know your brain. https://senmagazine.co.uk/articles/1096-how-your-brain-works-differently-as-a-dyslexic.html (Accessed January 2020). (2020).

46. Zambo, D. Using Qualitative Methods to Understand the Educational Experiences of Students with Dyslexia. The Qualitative Report, 9(1), 80-93. Retrieved from https://nsuworks.nova.edu/tqr/vol9/iss1/5 (2004).

47. W3C. (2018a), “Accessibility”, available at: https://www.w3.org/standards/webdesign/accessibility (accessed 26 November 2020).

48. W3C. (2018b), "Web Content Accessibility Guidelines (WCAG) 2.1", available at: https://www.w3.org/TR/WCAG21/ (accessed 26 November 2020).

49. W3C. (2020), "Understanding Success Criterion 3.3.7: Accessible Authentication", available at: https://www.w3.org/WAI/WCAG22/Understanding/accessible-authentication (accessed 26 November 2020).

50. GOV.UK. (2020), "Making your service accessible: an introduction", GOV.UK, available at: https://www.gov.uk/service-manual/helping-people-to-use-your-service/making-your-service-accessible-an-introduction (accessed 26 November 2020).

51. UK Home Office. (2016), "Dos and don'ts on designing for accessibility - Accessibility in government", available at: https://accessibility.blog.gov.uk/2016/o9/02/dos-and-donts-on-designing-for-accessibility/ (accessed 26 November 2020).

52. Dyslexia Scotland. (2015), "Dyslexia and ICT”, available at: https://www.dyslexiascotland.org.uk/our-leaflets (accessed 26 November 2020).

53. W3C. (2020), "G218: Email link authentication", available at: https://www.w3.org/WAI/WCAG22/Techniques/general/G218 (accessed 26 November 2020). 